\title{
FERROMAGNETIC FLOW OF VISCOUS FLUID IN A SLOT BETWEEN FIXED SURFACES OF REVOLUTION
}

\begin{abstract}
In this paper the steady laminar flow of viscous incompressible ferromagnetic fluid is considered in a slot between fixed surfaces of revolution having a common axis of symmetry. The boundary layer ferromagnetic equations for axial symmetry are expressed in terms of the intrinsic curvilinear orthogonal coordinate system $x, \theta, y$.The method of perturbation is used to solve the boundary layer equations. As a result, the formulae defining such parameters of the flow as the velocity components $v_{x^{\prime}}, v_{y}$ and the pressure, were obtained.
\end{abstract}

Keywords: laminar flow, incompressible ferromagnetic fluid, method of perturbation

\section{INTRODUCTION}

Magnetic fluids are a group of materials with properties hardly ever encountered in nature $[9,15]$. A combination of fluid and ferromagnetic material makes, without external magnetic field, magnetic fluids not to show magnetization and, reversely, the magnetic fluid, when subjected to external magnetic field, changes its properties $[3,8,10]$. Depending on the concentration of ferromagnetic particles, the magnetic fluid behaves like Newton fluid or non-Newton fluid [10].

Nowadays there are many different applications of magnetic fluids in engineering, e.g., in slide bearings, clutches, brakes, seals, electrohydraulic servomotors, linear and rotary mufflers, etc $[1,9,11,13]$.

Modeling of laminar flow of viscous fluids going through slots between movable and non-movable rotating surfaces exposed to magnetic field still plays a significant role in engineering $[6,7,12,14,16]$. With that in mind, it is important for the studies in this field to be performed both in terms of theory and possible practical applications $[2,4,5]$.

The purpose of this study is to analyze the theory of the impact of magnetic field and the flow inertia on the ferromagnetic fluid flow in a slot between fixed surfaces of revolution.

\section{BASIC EQUATIONS}

The motion of the ferromagnetic fluid in the slot, as shown in Fig. 1, is laminar and isothermal.

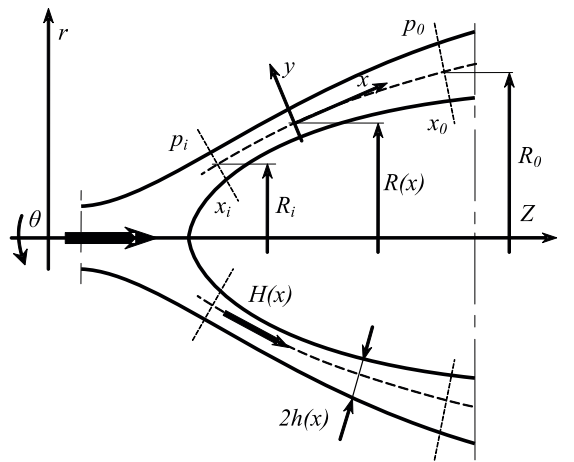

Fig. 1. Ferromagnetic fluid flow area

The flow takes place in the external, steady and heterogeneous magnetic field. However, it has been assumed that the ferromagnetic fluid is electrically non-conductive and the vectors of magnetic field strength and magnetization, respectively, are parallel to each other. The surfaces are 
described with the function which denotes the radius of the median between the fixed surfaces, together with the function which denotes the distance of each surface from the median, measured along the normal to the median.

According to the principles of mass momentum conservation, the equations of motion are [3]:

The continuity equation:

$$
\nabla \boldsymbol{V}=0
$$

The momentum equation:

$$
\rho(\boldsymbol{V} \nabla) \boldsymbol{V}=-\nabla p+\mu \Delta \boldsymbol{V}+\mu_{0}(\boldsymbol{M} \nabla) \boldsymbol{H}
$$

where: $\boldsymbol{V}, \boldsymbol{M}, \boldsymbol{H}, p, \rho, \mu, \mu_{0}$ - are: the velocity vector of fluid flow, the vector magnetization vector of the magnetic field, pressure, density, dynamic viscosity, and magnetic permeability of vacuum.

Eqs (1) and (2), to be completed, require some additional equations describing the magnetic field, i.e. the magnetostatic equations of:

$$
\begin{gathered}
\nabla \times \boldsymbol{H}=0 \\
\nabla \boldsymbol{B}=0 \\
\boldsymbol{B}=\mu_{0}(\boldsymbol{H}+\boldsymbol{M})
\end{gathered}
$$

The equations for motion are assumed to describe the general case of the flow in the curvilinear coordinates system $[14,16]$. If the asymptotic transformations have been made, the equations can be reduced to a simpler form:

$$
\begin{gathered}
\frac{1}{R} \frac{\partial\left(R v_{x}\right)}{\partial x}+\frac{\partial v_{y}}{\partial y}=0 \\
\rho\left(v_{x} \frac{\partial v_{x}}{\partial x}+v_{z} \frac{\partial v_{x}}{\partial y}\right)=-\frac{\partial p}{\partial x}+\mu \frac{\partial^{2} v_{x}}{\partial y^{2}}+\mu_{0} M_{x} \frac{\partial H_{x}}{\partial x} \\
\frac{\partial p}{\partial y}=0
\end{gathered}
$$

where: $v_{x}, v_{\theta}, v_{y}, p, M_{x}, H_{x}$ - respectively: velocity components, pressure, component of magnetization, component of magnetic field.

Thus, from equation (8) we have:

$$
p=p(x)
$$

The boundary conditions for velocity components are:

$$
v_{x}(x, \mp h)=0, \quad v_{y}(x, \mp h)=0 \text {, }
$$

Moreover, the inlet and outlet pressure under slot conditions can take the form of: for

for

$$
p=p_{i} \quad \text { for } \quad x=x_{i}
$$

$$
p=p_{0} \quad \text { for } \quad x=x_{0}
$$

where: $x_{i}$-denotes the inlet coordinate, $x_{0}$ - the outlet coordinate.

\section{SOLUTION OF THE EQUATIONS OF MOTION}

Introducing the following dimensionless quantities:

$$
\begin{aligned}
& \tilde{x}=\frac{x}{R_{o}}, \quad \tilde{y}=\frac{y}{h_{o}}, \quad \tilde{R}=\frac{R}{R_{o}}, \\
& \tilde{v}_{x}=\frac{v_{x}}{v_{o}}, \quad \tilde{v}_{y}=\frac{v_{y}}{v_{o}} \frac{R_{o}}{h_{o}}, \quad \tilde{p}=\frac{p h_{o}}{\mu v_{o}} \frac{h_{o}}{R_{o}}
\end{aligned}
$$

We can present the equations of motion (6) (8) in the form:

$$
\begin{gathered}
\frac{1}{\tilde{R}} \frac{\partial\left(\widetilde{\rho}_{e} R \tilde{v}_{x}\right)}{\partial \tilde{x}}+\frac{\partial\left(\widetilde{\rho}_{e} \tilde{v}_{y}\right)}{\partial \tilde{y}}=0 \\
\lambda\left(\tilde{v}_{x} \frac{\partial \tilde{v}_{x}}{\partial \tilde{x}}+v_{y} \frac{\partial \tilde{v}_{x}}{\partial \tilde{y}}\right)=-\frac{\partial \tilde{p}}{\partial \tilde{x}}+\frac{\partial^{2} \tilde{v}_{x}}{\partial \tilde{y}^{2}}+R F \widetilde{M}_{x} \frac{\partial \widetilde{H}_{x}}{\partial \tilde{x}}(14)
\end{gathered}
$$

$$
0=-\frac{\partial \tilde{p}}{\partial \tilde{y}}
$$

where:

$$
\begin{aligned}
& R F=\frac{\mu_{0} M_{0} H_{0} h_{0}^{2}}{\mu v_{0} R_{0}}, \quad \widetilde{H}_{x}=\frac{H_{x}}{H_{o}}, \quad \widetilde{M}_{x}=\frac{M_{x}}{M_{o}}, \\
& \lambda=R e \frac{h_{0}}{R_{o}}, \quad R e=\frac{2 \rho v_{0} h_{0}}{\mu}
\end{aligned}
$$

The quantities marked with subscript ,zero' are average values within the discussed flow domain; $\lambda$ - the modified Reynolds number which satisfied the condition:

$$
\lambda<1
$$

From Eq.(13) and (15) one can see that in the motion of ferromagnetic fluid, if condition (16) is satisfied, is a small parameter in Eq. (14).

Thus, the solution can be sought to in the form of power series with respect to $\lambda[16]$ :

$$
\begin{gathered}
\tilde{v}_{x}=\sum_{i=0}^{\infty} \lambda^{i} \tilde{v}_{x,}^{i} \quad \tilde{v}_{y}=\sum_{i=0}^{\infty} \lambda^{i} \tilde{v}_{y,}^{i} \\
\tilde{p}=\sum_{i=0}^{\infty} \lambda^{i} \tilde{p}_{,}^{i}
\end{gathered}
$$


Introducing the series (17) into Eq. (13) and (14), after necessary transformations, the terms referring to the same power of dimensional form $\lambda$, if we restrict ourselves to the linear approximation and return to the previous one, we receive the following equations:

$$
\begin{gathered}
\frac{1}{R} \frac{\partial\left(R v_{x}^{0}\right)}{\partial x}+\frac{\partial v_{y}^{0}}{\partial y}=0 \\
0=-\frac{\partial p^{0}}{\partial x}+\mu \frac{\partial^{2} v_{x}^{0}}{\partial y^{2}}+\mu_{0} M_{x} \frac{\partial H_{x}}{\partial x} \\
\frac{1}{R} \frac{\partial\left(R v_{x}^{1}\right)}{\partial x}+\frac{\partial v_{y}^{1}}{\partial y}=0
\end{gathered}
$$

$\rho\left[v_{x}^{0} \frac{\partial v_{x}^{0}}{\partial x}+v_{y}^{0} \frac{\partial v_{x}^{0}}{\partial y}\right]=-\frac{\partial p^{1}}{\partial x}+\mu \frac{\partial^{2} v_{x}^{1}}{\partial y^{2}}+\mu_{0} M_{x} \frac{\partial H_{x}}{\partial x}$

The boundary conditions, according to Eq.(10) and (11), take the form of [16]: for

$$
v_{x}^{0}=v_{x}^{1}=v_{y}^{0}=v_{y}^{1}=0 \text { for } y=\mp h
$$

for

$$
\begin{aligned}
& p^{0}=p_{i} \quad p^{1}=0 \text { for } x=x_{i} \\
& p^{0}=p_{0} \quad p^{1}=0 \text { for } x=x_{0}
\end{aligned}
$$

Integrating Eq. (18) and (21) and making the boundary conditions (22) and (23) to be valid, we have:

$$
\begin{gathered}
v_{x}^{0}=\frac{1}{2 \mu R h^{3}} \frac{p_{i}-p_{0}-B_{i}+B_{0}}{A_{i}-A_{0}}\left(y^{2}-h^{2}\right) \\
v_{y}^{0}=\frac{h^{\prime}}{2 \mu R h^{4}} \frac{p_{i}-p_{0}-B_{i}+B_{0}}{A_{i}-A_{0}}\left(h^{2} y-y^{3}\right) \\
p^{0}=B(x)+\frac{\left[A(x)-A_{0}\right]\left(p_{i}-B_{i}\right)-\left[A(x)-A_{i}\right]\left(p_{0}-B_{0}\right)}{A_{i}-A_{0}}
\end{gathered}
$$

$$
\begin{array}{r}
v_{x}^{1}=\frac{\rho\left(\frac{p_{i}-p_{0}-B_{i}+B_{0}}{A_{i}-A_{0}}\right)^{2}(R h)^{\prime}}{840 \mu^{3} R^{3} h^{7}}\left(35 h^{2} y^{4}-7 y^{6}+5 h^{6}-33 h^{4} y^{2}\right) \\
\begin{array}{r}
v_{y}^{1}=\frac{\rho\left(\frac{p_{i}-p_{0}-B_{i}+B_{0}}{A_{i}-A_{0}}\right)^{2}}{840 \mu^{3} h^{7}}\left\{\left[\frac{(R h)^{\prime}}{R^{2} h^{7}}\right]^{\prime}\left(y^{7}-7 h^{2} y^{5}-5 h^{6} y+11 h^{4} y^{3}\right)+\right. \\
\left.+\frac{(R h)^{\prime} h^{\prime}}{R^{2} h^{7}}\left(44 h^{3} y^{3}-14 h y^{5}-30 h^{5} y\right)\right\}
\end{array}
\end{array}
$$$$
p^{1}=D(x)-\frac{\left[A(x)-A_{0}\right] D_{i}-\left[A(x)-A_{i}\right] D_{0}}{A_{i}-A_{0}}
$$

where:

$$
\begin{aligned}
& R^{\prime}=\frac{d R}{d x}, \quad h^{\prime}=\frac{d h}{d x}, \\
& A(x)=\int \frac{d x}{R(x) h^{3}}, \quad A_{i}=A\left(x_{i}\right), \quad A_{0}=A\left(x_{0} .\right. \\
& B(x)=\frac{\mu_{0} M_{0} H_{0} R_{0}}{R}, \quad B_{i}=B\left(x_{i}\right), \quad B_{0}=B\left(x_{\mathrm{C}}\right.
\end{aligned}
$$

The complete solution of the ferromagnetic fluid flow problem inside a slot between curvilinear surfaces is made up of the sum of partial solutions.

\section{FERROMAGNETIC FLUID FLOW BETWEEN FIXED CONICAL SURFACES}

After introducing the functions used for describing the flow area geometry (Fig. 2) in Eq. (24) through (29):

$$
\begin{aligned}
& R(x)=x \sin (\alpha), \quad R_{i}=x_{i} \sin (\alpha), \\
& R_{0}=x_{0} \sin (\alpha), \quad R^{\prime}=\sin (\alpha)
\end{aligned}
$$

and introducing the dimensionless quantities:

$$
\begin{array}{ll}
\xi=\frac{x}{R_{0}}, & \eta=\frac{y}{h}, \quad \bar{R}^{\prime}=1, \\
\bar{v}_{x}=\frac{v_{x}}{v_{x \max }^{0}}, \quad \bar{v}_{y}=\frac{v_{y}}{v_{x \max }^{0}} \frac{R_{0}}{h}, \quad v_{x \max }^{0}=\frac{p_{0} h^{2}\left(\bar{p}_{i}-1\right)}{2 x \mu R_{0}\left(a_{0}-a_{i}\right)}
\end{array}
$$

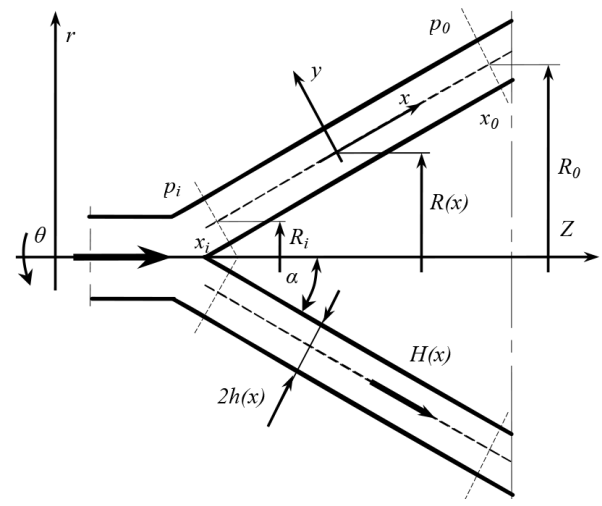

Fig. 2. Conical slot geometry

we get the following dimensionless formulas representing the velocity and pressure field in the slot:

$$
\bar{v}_{x}^{0}=\left(1-\frac{\bar{B}_{i}-\bar{B}_{0}}{\bar{p}_{i}-1}\right)\left(1-\eta^{2}\right),
$$

$$
\bar{v}_{y}^{0}=0
$$

$$
\bar{p}^{0}=\bar{B}(x)+\frac{\left[a(x)-a_{0}\right]\left(\bar{p}_{i}-\bar{B}_{i}\right)-\left[a(x)-a_{i}\right]\left(1-\bar{B}_{0}\right)}{a_{i}-a_{0}},
$$




$$
\begin{gathered}
\bar{v}_{x}^{1}=-\frac{1}{420 \xi^{2}} \wedge c \frac{a_{i}-a_{0}}{\bar{p}_{i}-1} \frac{\left[\bar{p}_{i}-1-\bar{B}_{i}+\bar{B}_{0}\right]^{2}}{a_{i}-a_{0}}\left(5-7 \eta^{6}+35 \eta^{4}-33 \eta^{2}\right) \\
\bar{v}_{y}^{1}=\frac{1}{240 \xi^{3}} \wedge c \frac{a_{i}-a_{0}}{\bar{p}_{i}-1}\left[\bar{p}_{i}-1-\bar{B}_{i}+\bar{B}_{0}\right]^{2}\left(\eta^{7}-7 \eta^{5}+11 \eta^{3}-5 \eta\right) \\
\bar{p}^{-1}=\bar{D}(x)-\frac{\left[a(x)-a_{0}\right] \bar{D}_{i}-\left[a(x)-a_{i}\right] \bar{D}_{0}}{a_{i}-a_{0}}
\end{gathered}
$$

where:

$$
\begin{aligned}
& a(x)=\ln (\xi), \quad a_{i}=a\left(\xi_{i}\right), \quad a_{0}=a\left(\xi_{0}\right), \\
& \bar{B}(x)=\frac{\mathrm{RF}}{\xi}, \quad \bar{B}_{i}=\bar{B}\left(\xi_{i}\right), \quad \bar{B}_{0}=\bar{B}\left(\xi_{0}\right), \\
& \mathrm{RF}=\frac{\mu_{0} M_{0} H_{0}}{p_{0}}, \\
& \bar{D}(x)=-\frac{3}{35} \wedge c \frac{\left(\bar{p}_{i}-1-\bar{B}_{i}+\bar{B}_{0}\right)^{2}}{\xi^{2}}, \quad \bar{D}_{i}=\bar{D}\left(\xi_{i}\right), \\
& \bar{D}_{0}=\bar{D}\left(\xi_{0}\right), \quad \wedge c=\frac{\rho p_{0} h^{4}}{\mu^{2} R_{0}^{2}\left(a_{i}-a_{0}\right)^{2}} .
\end{aligned}
$$

The above given formulas have been illustrated in Fig 3 Fig 6.

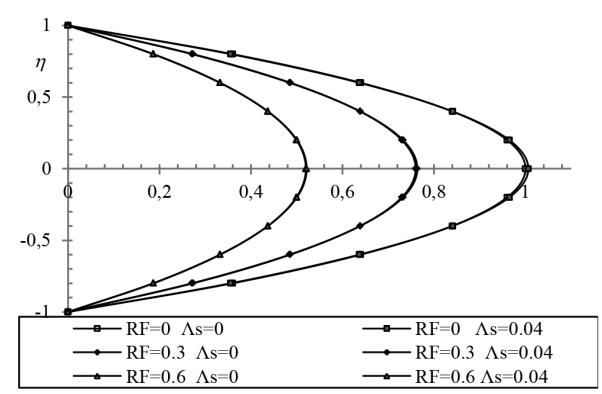

Fig. 3. Effect of the magnetic pressure number $R F$ and longitudinal inertia forces on the velocity profile $\bar{v}_{x}$ and $\bar{v}_{x}^{0},\left(\xi_{i}=0,2, \xi_{0}=0,6, \bar{p}_{i}=5,\right)$

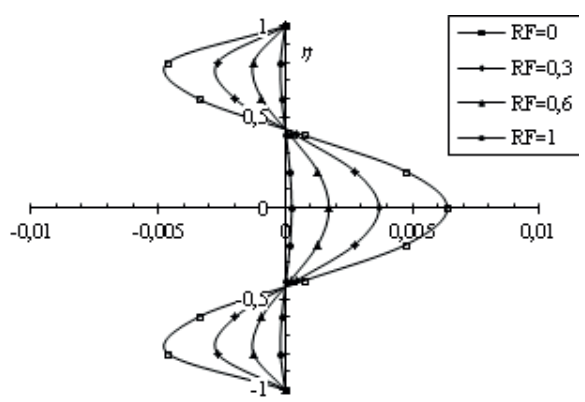

Fig. 4. Effect of the magnetic pressure number $R F$ and longitudinal inertia forces on the velocity profile $\bar{v}_{x}^{1},\left(\xi_{i}=0,2, \quad \xi_{0}=0,6, \bar{p}_{i}=5, \wedge c=0,04\right)$

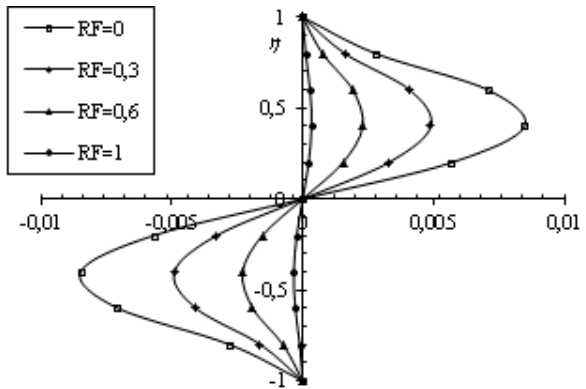

Fig. 5. Effect of the magnetic pressure number $R F$ and longitudinal inertia forces on the velocity profile $\bar{v}_{y}^{1},\left(\xi_{i}=0,2, \quad \xi_{0}=0,6, \bar{p}_{i}=6, \Lambda c=0,04\right)$,

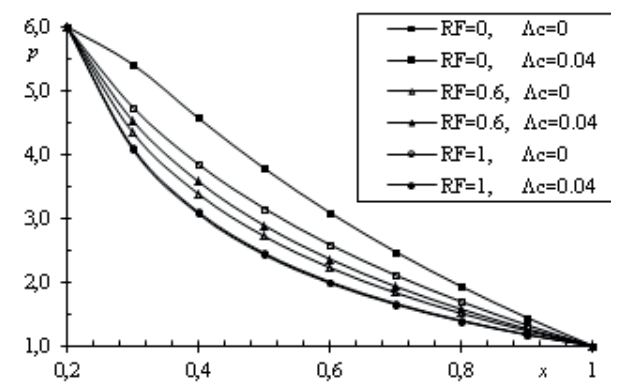

Fig. 6. Effect of the magnetic pressure number RF and dimensionless parameter corresponding to the effect of longitudinal inertia forces on the pressure $\bar{p}$. $\left(\xi_{i}=0,2, \quad \xi_{0}=1, \bar{p}_{i}=6\right)$

\section{FERROMAGNETIC FLUID FLOW BETWEEN FIXED SPHERICAL SURFACES}

The parameters used to describe the flow area geometry can be written as follows (Fig. 7):

$$
\begin{aligned}
& R(x)=R_{k} \sin (\varphi), \quad R_{i}=R_{k} \sin \left(\varphi_{i}\right), \\
& R_{0}=R_{k} \sin \left(\varphi_{0}\right), \\
& R^{\prime}=\cos (\varphi), \quad \varphi=\frac{x}{R_{k}} .
\end{aligned}
$$

After introducing the functions used for describing the geometry of the flow area (Fig. 7) in Eq. (24) through (29) and providing the dimensionless quantities:

$$
\begin{aligned}
& \eta=\frac{y}{h}, \quad \bar{R}=\frac{R(x)}{R_{0}}, \quad \bar{R}^{\prime}=\cos (\varphi) \\
& \bar{v}_{x}=\frac{v_{x}}{v_{x \max }^{0}}, \quad \bar{v}_{y}=\frac{v_{y}}{v_{x \max }^{0}} \frac{R_{k} \sin \left(\varphi_{0}\right)}{h}, \quad v_{x \max }^{0}=\frac{p_{0} h^{2}\left(\bar{p}_{i}-1\right)}{2 \mu \sin (\varphi)\left(a_{0}-a_{i}\right) R_{0}}
\end{aligned}
$$




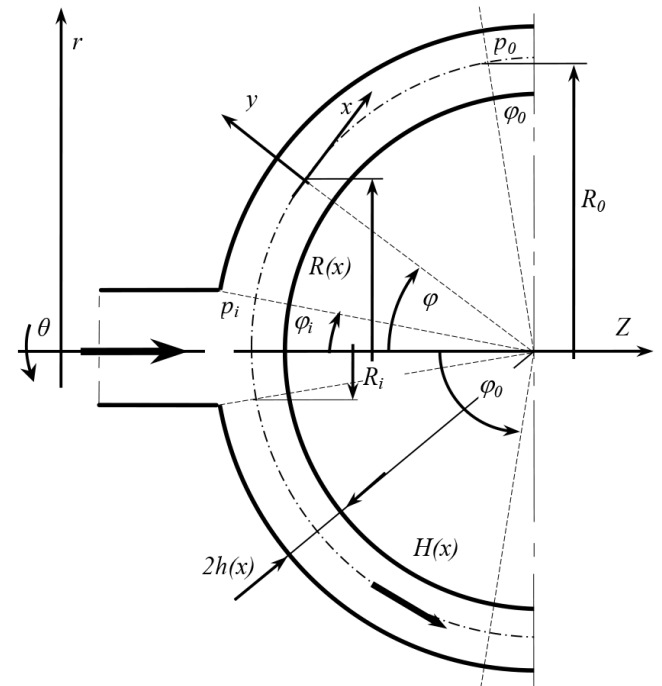

Fig. 7. Spherical slot geometry

we obtain the formulas representing the motion of the ferromagnetic fluid inside the slot between the fixed spherical surfaces:

$$
\begin{gathered}
\bar{v}_{x}^{0}=\left(1-\frac{\bar{B}_{i}-\bar{B}_{0}}{\bar{p}_{i}-1}\right)\left(1-\eta^{2}\right), \\
\bar{v}_{y}^{0}=0 \\
\bar{p}^{0}=\bar{B}(x)+\frac{\left[a(x)-a_{0}\right]\left(\bar{p}_{i}-\bar{B}_{i}\right)-\left[a(x)-a_{i}\right]\left(1-\bar{B}_{0}\right)}{a_{i}-a_{0}}, \\
\bar{v}_{x}^{1}=-\frac{1}{420} \wedge s \frac{a_{i}-a_{0}}{\bar{p}_{i}-1} \frac{\left[\bar{p}_{i}-1-\bar{B}_{i}+\bar{B}_{0}\right]^{2}}{a_{i}-a_{0}} \frac{\cos (\varphi)}{\sin ^{2}(\varphi)}\left(5-7 \eta^{6}+35 \eta^{4}-33 \eta^{2}\right) \\
\bar{v}_{y}^{1} \frac{1}{240 \sin ^{3}(\varphi)} \wedge s \frac{a_{i}-a_{0}}{\bar{p}_{i}-1}\left[\bar{p}_{i}-1-\bar{B}_{i}+\bar{B}_{0}\right]^{2} \frac{1+\cos ^{2}(\varphi)}{3}\left(\eta^{7}-7 \eta^{5}+11 \eta^{3}-5 \eta\right) \\
\bar{p}^{1}=\bar{D}(x)-\frac{\left[a(x)-a_{0}\right] \bar{D}_{i}-\left[a(x)-a_{i}\right] \bar{D}_{0}}{a_{i}-a_{0}}
\end{gathered}
$$

where:

$$
\begin{aligned}
& a(\varphi)=\ln \left|\tan \left(\frac{\varphi}{2}\right)\right|, \quad a_{i}=a\left(\varphi_{i}\right), \quad a_{0}=a\left(\varphi_{0}\right), \\
& \bar{B}(\varphi)=\frac{\mathrm{RF}}{\sin (\varphi)}, \quad \bar{B}_{i}=\bar{B}\left(\varphi_{i}\right), \quad \bar{B}_{0}=\bar{B}\left(\varphi_{0}\right), \\
& \mathrm{RF}=\frac{\mu_{0} M_{0} H_{0}}{p_{0}}, \\
& \bar{D}(x)=-\frac{3}{35} \wedge s \frac{\left(\bar{p}_{i}-1-\bar{B}_{i}+\bar{B}_{0}\right)^{2}}{\sin ^{2}(\varphi)}, \\
& \bar{D}_{i}=\bar{D}\left(\varphi_{i}\right), \quad \bar{D}_{0}=\bar{D}\left(\varphi_{0}\right), \quad \wedge s=\frac{\rho p_{0} h^{4}}{\mu^{2} R_{0}^{2}\left(a_{i}-a_{0}\right)^{2}} .
\end{aligned}
$$

Since diagrams of the ferromagnetic fluid flow velocity distribution along the slot between the fixed spherical surfaces show no substantial differences from the ferrofluid flow velocity distribution in the slot between the fixed conical surfaces, only the formulas for pressure profiles are illustrated in the diagram (Fig. 8).

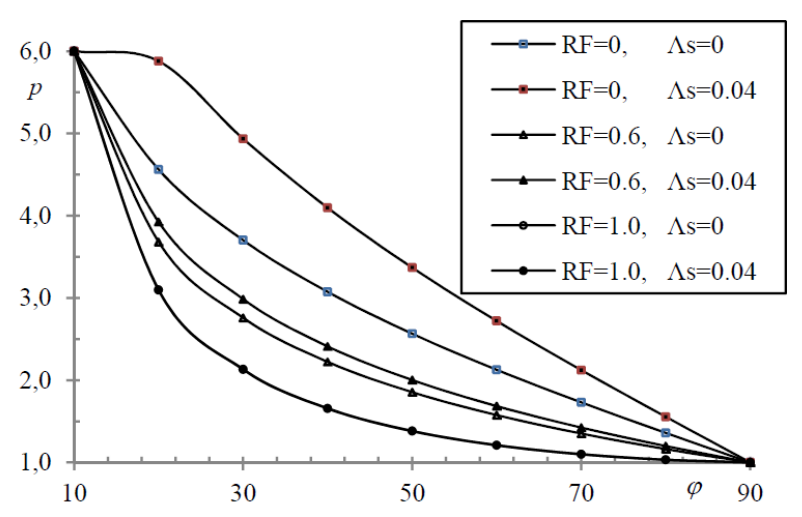

Fig. 8. Effect of the magnetic pressure number RF and dimensionless parameter corresponding to the effect of longitudinal inertia forces on the pressure $\bar{p}$ $\left(\varphi_{i}=10^{\circ}, \varphi_{0}=90^{\circ}, \bar{p}_{i}=6\right)$

\section{DISCUSSION OF THE RESULTS}

With the diagram and equations describing the components of the field of velocity and pressure, it is possible to formulate the following conclusions:

For longitudinal velocity $v_{x}$ (Fig. 3 ):

- the main profile of velocity $\mathrm{vx}$ is a parabolic profile identical to traditional Poisseuille flow profile caused by a steady gradient of pressure (constant pressure difference) characteristic for the so-called Reynolds approximation;

- the so-called secondary profile symmetric to the slot symmetry axis formed due to the occurrence of the flow inertia effects, overlaps the main velocity profile $v_{x}$ (Fig. 4). For transverse velocity $v_{y}$ (Fig. 5):

- distribution of transverse velocity $v_{y}$ is the result of inertia effects occurring in the flow,

- an increase in the magnetic field strength expressed with an increase in the value of magnetic pressure number $R$ results in decreasing the longitudinal and transverse velocity components.

For pressure (Fig. 6 and Fig. 8):

- inertia effects $\left(\Lambda_{L c}, \Lambda_{L s}>0\right)$ cause a minimum pressure increase along the slot,

- an increase in the magnetic field strength expressed with an increase in the value of magnetic pressure number, causes a pressure drop along the slot.

The flow through slots with curvilinear profiles is less susceptible to inertia effects. 


\section{BIBLIOGRAPHY}

1. Bednarek S.: Ferromagnetic fluids - materials with unusual properties and their applications, 2009, Foton, Institute of Physics, Jagiellonian University, spring 104

2. Buhler L.: Magnetohydrodynamic flow in ferromagnetic pipes, Forschungszentrum Karsruhe GmbH, 2002

3. Ezekiel F.D.: Ferrolubricants: 1975, New Application, Mechanical Engineering, 2

4. Huang W., Wang X.: Ferrofluids lubrication: a status report, 2015, Lubrication Science, 02

5. Lemarquand V. , Lemarquan G.: Ferrofluid seals, ,New Tribological Ways, 2011, 04.

6. McAlister K. W., Rice W.: Throughflows between rotating surfaces of revolution, having similarity solutions, J. Appl. Mech. Trans. ASME, Series E, 1970, 37, 4

7. McAlister K.W., Rice W.: Flows between stationary surfaces of revolution, having similarity solutions, J. Appl. Mech. Trans. ASME, Series E, 1972, 39, 2

8. Muc A., Barski M.: Magnetorheological fluids and their practical applications, Technical magazine, Cracow University of Technology , 2007, Z1-M

9. Nada G.S., Osman T.A.: Journal bearings lubricated by magnetic fluids with couple stresses, Tribology Letters, $2007,27,3$

10. Neuringer J.L., Rosenzweig R.E.: Ferrohydrodynamics, Phys. Fluids, 1964, 7, 12

11. Ravaud R., Lemarquand G., Lemarquand V.: Mechanical properties of ferrofluids applications: Centering effect and capacity of a seal, Tribology International , 2010, 01.

12. Reinhardt E., Lund W.J. : The influence of fluid inertia on the dynamic properties of journal bearings, J. Lubric. Technol., Trans. ASME, Series F , 1975,, 97, 2

13. Safar Z.S., Nada G.S., Osman T.A.: Static and dynamic characteristic of magnetized journal bearing lubricated with ferrofluids, Tribology International , 2001, 02

14. Sawicki J.,: Magnetohydromagnetic flow of viscous fluid in a slot between curvilinear surfaces of revolution, Engineering Transactions, PAN-IPPT, 1996, 44,1

15. Szliomis M.I.: Magnitnyye zhidkosti ( in Russian), Usp., Fiz. Nauk, 1974, 112, 3
16. Walicki E. , Sawicki J., Ziejewski M.: Inertia effect in magnetic throughflow fluid in a slot between fixed surfaces of revolution, Mec. Applique, 1978, 23, 6.

\section{CONTACT WITH THE AUTHOR}

Sawicki Jerzy, Assoc. Prof. UTP

e-mail: jerzy.sawicki@utp.edu.pl

University of Science and Technology in Bydgoszcz Faculty of Mechanical Engineering Department of Applied Mechanics

Al. Prof. S. Kaliskiego 7

85-796 Bydgoszcz

Poland 